\title{
Successful management of complicated multi-organ hydatid cysts
}

\begin{abstract}
We report herein a successful management of a case of 24-year-old unmarried male presented to us with the complicated left pulmonary hydatid cyst as left empyema thoracis who eventually developed further thoracic and abdominal complications after the surgical removal of one big hydatid cyst from left lung and two big hydatid cysts from both lobes of liver. The importance of unwearied approach of clinicians and successful applications of multi-modality conservative, interventional, medical and surgical procedures are also discussed for the best outcome of this subset of patients.
\end{abstract}

Keywords: Complicated lung hydatid cyst, liver hydatid disease, hydatid cyst, echinococcus granulosus, multi-organ hydatid, empyema, intestinal obstruction, bile leak, hydatidosis
Volume 7 Issue 5 - 2017

\author{
Pankaj Srivastava,' Arvind Kumar, ${ }^{2}$ Belal Bin \\ Asaf ${ }^{2}$ \\ 'Om Surgical Center \& Maternity Home, India \\ ${ }^{2}$ Sir Ganga Ram Hospital, India
}

\begin{abstract}
Correspondence: Pankaj Srivastava, Laparoscopic, Thoracic, Thoracoscopic \& VATS Surgeon, Om Surgical Center \& Maternity Home, SA I7/3, P-4, Sri Krishna Nagar, Paharia, Ghazipur Road,Varanasi, UP, India, Pin-221007, Tel +91-5422586191, Fax9.19415E+I I, Email drpankajbns@gmail.com
\end{abstract}

Received: July 20, 2017 | Published: September 19, 2017
Abbreviations: PHC, pulmonary hydatid Cyst; HHC, hepatic hydatid cyst; HPHC, hepato pulmonary hydatid cyst; ICD, intercostal drain; POD, post operative day

\section{Introduction}

Hydatid disease is a zoonotic disease, caused by Echinococcus granulosus, tend to involve liver, lung, brain, heart, bones and rarely kidneys and intestines. Lung is the second localization organ in adults after liver. ${ }^{1}$ Hepato-pulmonary hydatid cysts (HPHC), were reported up to $34.8 \%{ }^{2}$ The higher rate of complicated pulmonary hydatid cyst (PHC) were associated with hepato-pulmonary localization (50\%) than with single PHC localization. ${ }^{3}$ Multiple organ involvement including brain, heart, intestine and kidney was also reported with the only neurological symptoms. ${ }^{4}$ For PHCs, cystectomy and cystostomy with or without capitonnage are commonly applied operative procedures but some patients may require major lung resection. Although Hepatic hydatid cysts (HHC) can be treated by surgery, chemotherapy and/ or percutaneous aspiration, surgery remains the traditional and established treatment applicable over the entire spectrum of disease. ${ }^{5}$

\section{Case Presentation}

A 24-year-old unmarried, non-smoker, non-alcoholic male presented with chief complaints of breathlessness, fever, and abdominal pain of 2 months duration. He was investigated elsewhere and was found to have hydatid cysts of liver and lung. He went to tertiary care hospital where a percutaneous aspiration was tried. The procedure failed and he developed hydropneumothorax for which an ICD was placed. The patient could not get any relief and purulent drain was continuous then he came to us for further management.

On admission, oral temperature was $100^{\circ} \mathrm{F}$, pulse rate was $100 /$ minute, Blood Pressure was 120/80 mmHg, Respiratory rate was 30/ min. There was decreased air entry on left lung auscultation and one Intercostal drain (ICD) in situ on left side. Per abdomen examination was insignificant except hepatomegaly. Chest X-ray PA view after aspiration showed hydropneumothorax with clear-cut cyst margins on the left lower region with ICD inside (Figure 1). CECT abdomen and chest revealed two hydatid cysts in the right lobe of the liver (Figures $2 \& 3$ ). After all preoperative evaluation, patient was found fit for the surgery. His all hematologic and biochemical parameters were within normal limits. Therefore, it was planned to deal with the lung and liver hydatids at the same sitting under general anesthesia first with liver followed by lung hydatid cyst.

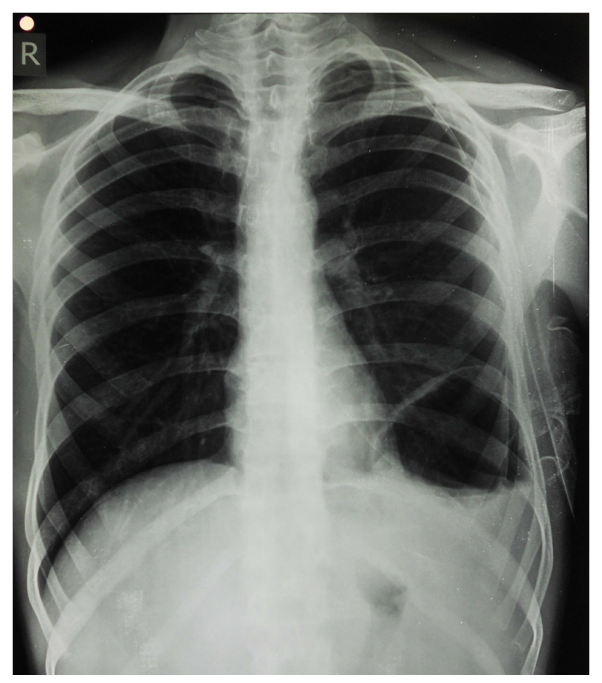

Figure I Chest X-ray PA View showing cyst wall with hydropneumothorax and ICDs in situ.

\section{Laparotomy}

After general anesthesia and intubation with single lumen endotracheal tube, the patient was put in supine position. The abdomen was approached by upper midline incision. Two large cysts were noted. One in left lobe and second one was in inferior surface of right lobe. There was no peritoneal hydatidosis. Rest of the peritoneal cavity was normal. Liver was mobilized by dividing the coronary ligament and left triangular ligament. The area around the liver was packed with povidone-iodine soaked abdominal pads to avoid peritoneal contamination. Left cyst was handled by using an Aaron's cone. The cyst was incised and fluid was evacuated. Cyst wall was deroofed. Membranes were evacuated. Cavity was filled with povidone-iodine and left for 4 to 5 minutes. The entire cavity was well 
cleaned and evacuated. The cavity was then meticulously inspected for biliary communication. The right lobe cyst was then handled in the similar manner. The gallbladder was found hanging between the two cysts; therefore, a cholecystectomy was also done. The omentum was mobilized and both the cavities were obliterated by omentum. One $28 \mathrm{Fr}$ drain was placed in Morrison's pouch. Abdomen was closed in layers after confirming hemostasis.

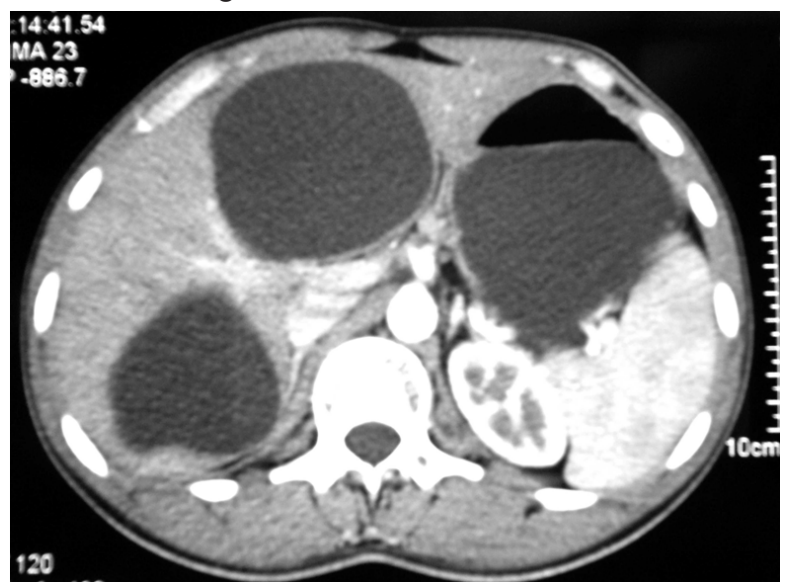

Figure 2 CECT Abdomen revealed hydatid cyst in both the lobes of liver.

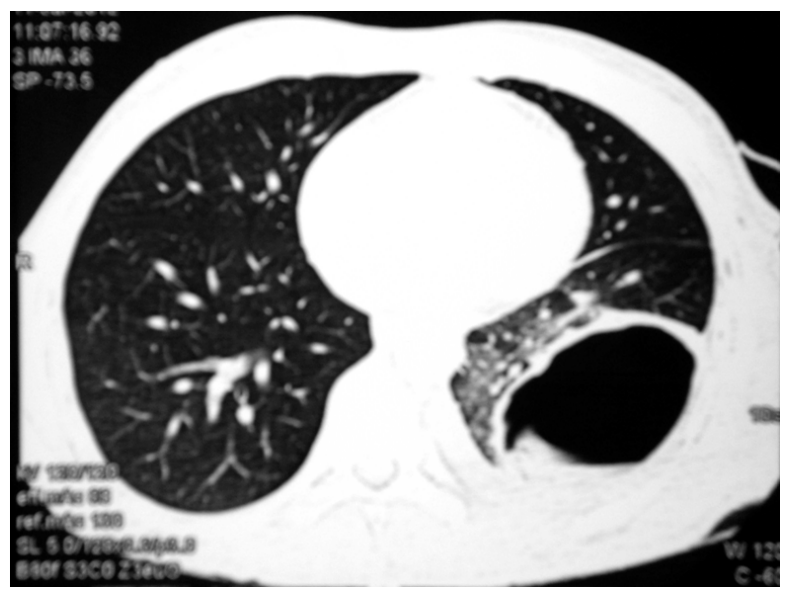

Figure 3 CECT Chest revealed ruptured hydatid cyst in left lower lobe.

\section{Thoracotomy}

After the laparotomy, the endotracheal tube was converted to double lumen tube. A left posterolateral (PL) thoracotomy was done and pleural cavity was entered through $5^{\text {th }}$ ICS. Left lung was collapsed. The entire chest cavity was filled with pus and thick pleural peel was present. All the purulent material was aspirated. Complete decortication was done with excision of damaged portion of left lower lobe (Figure 4). There was cavity in the lower lobe into which a bronchus was opening. That bronchus was closed with $3 / 0$ polypropylene using surgicel ${ }^{\circledR}$ as pledgets. A thorough wash was given with lukewarm normal saline. Hemostasis was secured. Two 28 Fr. ICDs were placed anteriorly and posteriorly to drain air and fluid, then chest was closed in layers. Cysts and the resected lung tissue were sent for histopathologic examination which had confirmed the clinical diagnosis of hydatid disease for both liver and lung cyst and fungal granulomatous inflammation in the cyst wall of lung hydatid.

\section{Post-operative course}

The ICDs showed air-leak on Post-operative day (POD) 1. To manage the air-leak ICDs were connected to medela ${ }^{\circledR}$ portable suction system. From POD-4 onwards, he started having bile leak in the abdominal drain. He was observed for 4 days expecting spontaneous resolution of bile-leak. But the drain output stabilized at $150-200 \mathrm{~mL} /$ day. The consultation was taken from gastroenterologist and ERCP was performed on POD 8 which revealed a leak from the right hepatic duct branch. Stent was placed across the leak. Post-ERCP the bile leak was increased to $600 \mathrm{~mL} /$ day and the patient developed fever $\left(101^{\circ} \mathrm{F}-102^{\circ} \mathrm{F}\right)$ and pain in abdomen. He was taken up for repeat ERCP on POD-10 and this time two plastic stents were placed across the leak. After second attempt of ERCP, the bile leak subsided and his abdominal drain out-put became nil after two days (POD-12).

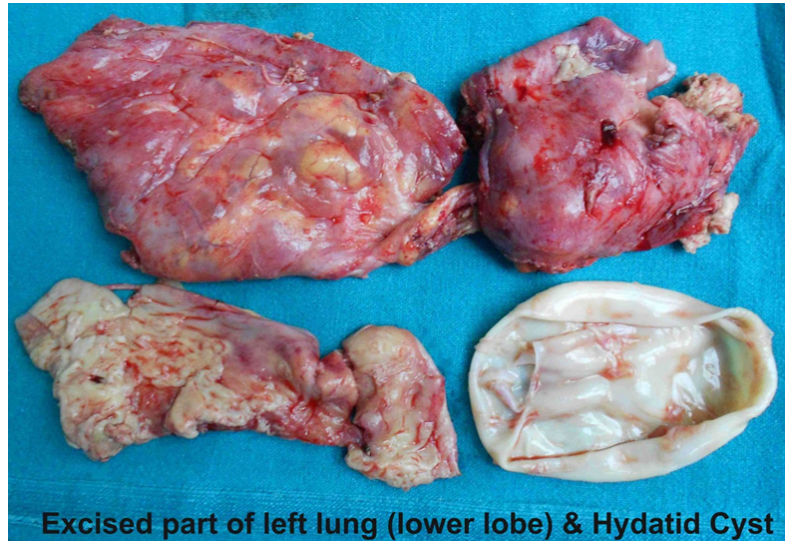

Figure 4 Excised parts of the lower lobe of left lung with hydatid cyst.

There was persistent and significant air-leak in the ICDs which prevented the left lung expansion. A bronchoscopy was done. The segments of the left lung were blocked one by one with bronchoscopic balloon checking for reduction in air-leak each time. Air-leak significantly reduced on blocking the lateral division of apical segment of left lower lobe. $2 \mathrm{~mL}$ of cyanoacrylate glue was injected into the subsegment. However the air-leak persisted even after the procedure. In the meanwhile, patient again developed fever $\left(101^{\circ} \mathrm{F}\right)$ and abdominal pain two days after repeat ERCP(POD-14). A USG abdomen was done which revealed significant collection in epigastrium, right subhepatic space, and between bowel loops. He was managed conservatively for 2 days but when the fever did not subside and collection did not resolve (as confirmed on repeat USG); consultation taken from interventional radiologist who did percutaneous catheter drainage of septated right hepatic collection. Approximately $80 \mathrm{~mL}$ of thick pus was drained. The epigastric collection was relatively anechoic and $30 \mathrm{~mL}$ of bile aspirated from that side. Both were sent for culture-sensitivity which revealed Candida tropicalis sensitive to amphotericin B which was started immediately. Patient fever was settled after catheter drainage of collection. He was well for 4 days after this procedure (POD-20). Air-leak in the ICD also started decreasing. Once the percutaneous catheter and abdominal drain output became nil, a repeat USG abdomen was done which showed significantly reduced collections. Both the abdominal drains were subsequently removed. Patient was well for 3 days thereafter (POD-23) and allowed liquids and soft diet. On POD-27, patient started having pain abdomen and distension. $\mathrm{X}$-ray abdomen revealed multiple air-fluid level suggestive of acute intestinal obstruction (Figure 5). A CECT of chest and abdomen were done, left lung was found to have expanded significantly and there was minimal hydropneumothorax whereas abdomen revealed grossly dilated small bowel and large bowel loops with a pelvic collection. He was then taken up for exploratory laparotomy on POD-29. 


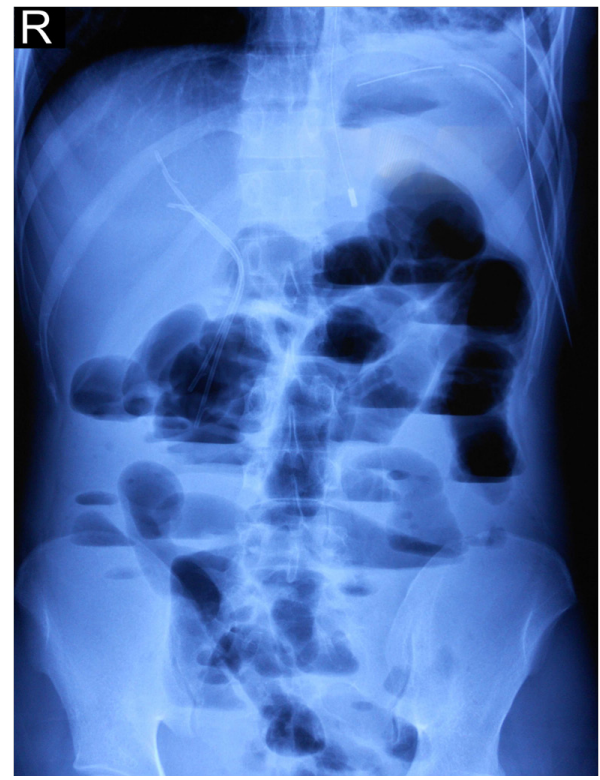

Figure 5 Multiple fluid-air levels with biliary stents in situ.

\section{Exploratory Laparotomy \& Thoracic window operation}

Second-look laparotomy was done through previous upper midline incision with below umbilical extension. Dilated small bowel loops were found. Loop of terminal ileum was found stuck in the pelvis. A thick and viscid pelvic collection was drained and adhesiolysis was done. Peritoneal cavity was thoroughly washed with copious amount of lukewarm normal saline. One 24 Fr Pelvic drain was placed and abdomen was closed in layers. Left thoracic cavity was approached by the previous PL thoracotomy incision, 2 ribs were resected and a window thoracostomy was done to manage the air-leak. The cavity was packed with povidone-iodine-soaked pads. Post-operatively, the patient was kept nil orally for 5 days and started on total parenteral nutrition (TPN) via central line. His bowel sounds returned and he passed flatus on POD-4. The Ryle's tube and abdominal drain were removed on POD-5. Daily dressing of thoracostomy wound was done and it showed satisfactory healing. Patient was gradually started on liquid diet from POD-6. By POD-10, he was able to take soft diet. The patient recovered well after the second surgery. Skin sutures were removed on POD-12. At the time of discharge (POD-15), patient is healthy with normal vitals and able to take normal diet. The thoracostomy wound is also healthy and started contracting.

\section{Discussion}

A synchronized hepatic and pulmonary hydatid cyst should be evaluated as a morbidity factor. The most common complications of the hepatic hydatid disease are infection and rupture of the cyst into the biliary tree.5 The most frequently isolated microbe is $E$. coli. Leakage of the cyst is essential to infection since the laminated membrane is a potent antibacterial barrier. Rupture of the hepatic hydatid cyst is an unusual complication owing to trauma or relentless expansion of the cyst causing pressure necrosis with resultant rupture into the peritoneal cavity, the pleural cavity or the bile duct. PHCs may remain asymptomatic for long time as elastic capability of the lung without any resistance give leverage for growth. Complications include super-infection, bacterial and fungal as well. ${ }^{6}$ and rupturespontaneous or iatrogenic (FNAC or Percutaneous aspiration). Surgery is still the treatment of choice which includes simple cystectomy, pleural cavity washings, and major decortication to lung resection. Post-operative complications are usually wound infection, prolonged air leak which we faced in our case that lead ultimately to window operation and hemorrhage. Biliary leakage in our case was well managed conservatively by stenting. Bile duct stenting must be considered in the beginning of the bile-leak to avoid biliary peritonitis, adhesions and unfortunate intestinal obstruction which occurred in our case for which we had to do exploratory laparotomy again. Window thoracostomy was the last resort for us to manage the persistent air-leak and to maintain the expansion of the lung. In these patients, thoracoplasty can also be considered to resolve the problem once infection got settled.

Conclusively, hydatid cyst should be considered in the differential diagnosis of uncertain chest pathologies, especially in rural areas where disease in endemic due to heavy animal handling. Though, there is controversy regarding the aspiration in the diagnosis of lung cystic lesion considering chances of the rupture of the cyst, anaphylaxis and dissemination, one should try to explore other noninvasive tools to make the final diagnosis of hydatid cyst of lung. High index of suspicion for cystic hydatidosis should be considered in any cystic mass for the prompt and correct diagnosis and successful management of the patient whether complicated or not. Purpose of reporting the case is to make clinicians aware of spectrum of complications associated with hepato-pulmonary hydatid disease and how to manage these complications successfully by multi-disciplinary and multi-modality approach.

\section{Acknowledgments}

None.

\section{Funding}

None.

\section{Conflicts of interest}

The authors declare that there is no conflict of interest.

\section{References}

1. Kilani T, Hammami S. Pulmonary hydatid and other lung parasitic infections. Curr Opin Pulm Med. 2002;8(3):218-223.

2. Aribas K, Kanat F, Tü rk E. Comparison between pulmonary and hepatopulmonary hydatidosis. Eur $J$ Cardiothorac Surg. 2002;21(3):489-496.

3. Arinc S, Kosif A, Ertugrul M, et al. Evaluation of pulmonary hydatid cyst cases. Int J of Surg. 2009;7(3):192-195.

4. Sabouni F, Ferdosian F, Mamishi S, et al. Multiple Organ Involvement with hydatid cyst. Iranian J Parasitol. 2010;5(2):65-70.

5. Malik AA, Bari SUL, Amin R, et al. Surgical management of complicated hydatid cysts of the liver. World J Gastrointest Surg. 2010;2(3):78-84.

6. Garcia MB, Lledias JP, Perez IG, et al. Primary Super-Infection of Hydatid Cyst-Clinical setting and Microbiology in 37 Cases. Am J Trop Med Hyg. 2010;82(3):376-378. 